\title{
Psychological effects of Anatomy dissections among pre-clinical medical students in Rajarata University of Sri Lanka
}

Rodrigo $\mathrm{PWDCH}^{1}$, Lasanthi SS ${ }^{1}$, Vijithananda $\mathrm{HHTSM}^{1}$, Dissanayake $\mathrm{CS}^{1}$, Sanjeewa WA $^{1}$, Warnasekara YPJN ${ }^{2}$, Koralegedara KIS ${ }^{1}$, Paththinige CS $^{1}$

${ }^{I}$ Department of Anatomy, Faculty of Medicine and Allied Sciences, Rajarata University of Sri Lanka.

${ }^{2}$ Department of Community Medicine, Faculty of Medicine and Allied Sciences, Rajarata University of Sri Lanka.

*chathurikarodrigo5@gmail.com

Anatomy teaching/learning in medical schools is frequently based around the use of human cadavers. Dissections are associated with multiple stresses including psychological effects. This study assessed the psychological impact of anatomy dissections among pre-clinical students of Faculty of Medicine and Allied Sciences, Rajarata University of Sri Lanka (FMAS, RUSL). A descriptive cross-sectional study was conducted among preclinical students in the first and second-year in FMAS, RUSL. The data was collected using a pilot-tested, self-administered questionnaire, and was analyzed using descriptive statistics and Chi-square test. The study was approved by Ethics Review Committee, FMAS, RUSL. Total of 305 of preclinical students [158 (51.8\%) first-year and 147 (48.2\%) second-year students] participated. Majority (68.9\%) were females. Factors that made students uncomfortable most during dissections were formaldehyde smell (47.2\%) and infrastructure of the dissection lab (16.4\%). Psychological effects of dissections included sleeping problems (2\%), learning difficulties (5.9\%), nightmares $(3.9 \%)$ and stress $(6.9 \%)$. Learning difficulties following exposure to cadavers were significantly more common among first-year students $(10.1 \%$ vs. $1.4 \%$; $<<0.001)$. No significant sex difference was observed. The main complains following dissection were reduced energy, diminished appetite and loss of interest and enjoyment (24.0\% vs. $11.6 \%, 14.6 \%$ vs. $17.0 \%, 14.6 \%$ vs. $10.9 \%$ respectively among first-year and second-year students). Depressed mood was reported by $15.8 \%$ of firstyear students and $8.8 \%$ of second-year students. Multiple psychological effects are shown to be associated with anatomy dissections. Identification and appropriate management of these stresses are important for enhancement of students' learning experience in Anatomy laboratory. 